\title{
Pc2-3 geomagnetic pulsations on the ground, in the ionosphere, and in the magnetosphere: MM100, CHAMP, and THEMIS observations
}

\author{
N. Yagova ${ }^{1}$, B. Heilig ${ }^{2}$, and E. Fedorov ${ }^{1}$ \\ ${ }^{1}$ Schmidt Institute of Physics of the Earth, Moscow, Russia \\ ${ }^{2}$ Tihany Geophysical Observatory MFGI, Tihany, Hungary \\ Correspondence to: N. Yagova (nyagova@yandex.ru)
}

Received: 23 April 2014 - Revised: 2 December 2014 - Accepted: 16 December 2014 - Published: 28 January 2015

\begin{abstract}
We analyze Pc2-3 pulsations recorded by the CHAMP (CHAllenging Minisatellite Payload) satellite in the F layer of the Earth's ionosphere, on the ground, and in the magnetosphere during quiet geomagnetic conditions. The spectra of Pc2-3 pulsations recorded in the F layer are enriched with frequencies above $50 \mathrm{mHz}$ in comparison to the ground Pc2-3 spectra. These frequencies are higher than the fundamental harmonics of the field line resonances in the magnetosphere. High quality signals with dominant frequencies $70-200 \mathrm{mHz}$ are a regular phenomenon in the $\mathrm{F}$ layer and in the magnetosphere. The mean latitude of the maximum Pc2-3 occurrence rate lies at $L \approx 3.5$ in the $\mathrm{F}$ layer, i.e., inside the plasmasphere. Day-to-day variations of the $L$ value of the CHAMP Pc2-3 occurrence rate maximum follow the plasmapause day-to-day variations. Polarization and amplitude of Pc2-3s in the magnetosphere, in the ionosphere, and on the ground allow us to suggest that they are generated as fast magnetosonic (FMS) waves in the outer magnetosphere and are partly converted into shear Alfven waves near the plasmapause. The observed ground-to-ionosphere amplitude ratio during the night is interpreted as a result of the Alfven wave transmission through the ionosphere. The problem of wave transmission through the ionosphere is solved theoretically by means of a numerical solution of the full-wave equation for the Alfven wave reflection from and transmission through a horizontally stratified ionosphere. The best agreement between the calculated and measured values of the ground-to-ionosphere amplitude ratio is found for $k=$ $5 \times 10^{-3} \mathrm{~km}^{-1}$, i.e., the observed ground-to-ionosphere amplitude ratio corresponds to a wave spatial scale which could provide a Doppler shift within a few percent of the apparent frequency of the Pc2-3 pulsations as recorded by a loworbiting spacecraft.
\end{abstract}

Keywords. Ionosphere (wave propagation) - magnetospheric physics (MHD waves and instabilities)

\section{Introduction}

The main morphological features of ground pulsations in the frequency range from $20 \mathrm{mHz}$ to $1 \mathrm{~Hz}$ (Pc1-3) were calculated almost fifty years ago (Guglielmi and Troitskaya, 1969). The most important difference between Pc3s and Pc1s is that Pc3 spectra are controlled mostly by extra-magnetospheric parameters while for Pc1, intramagnetospheric control dominates. A quasi-monochromatic source for both Pc3 and Pc1 is the ion cyclotron instability. The difference in central frequencies results from the different magnitudes of the main magnetic field in the foreshock and in the magnetosphere. Additional maxima in the observed pulsation spectra occur due to Alfven resonances in the magnetosphere-ionosphere system. The frequencies of mid-latitude Pc3s correspond to the frequencies of the first harmonics of the magnetospheric Alfven resonator at the corresponding $L$ shells and for Pc1s they coincide with the first harmonics of the ionospheric Alfven resonator (IAR).

Pulsations in the intermediate frequency range, Pc2 (80$300 \mathrm{mHz}$ ) are not often observed on the ground surface at middle and low geomagnetic latitudes $\left(\Phi<60^{\circ}\right)$. This frequency range corresponds to higher harmonics of the magnetospheric Alfven resonator and to the ion cyclotron resonance of heavy ions in the outer magnetosphere (Fraser et al., 1992).

Pulsations with frequencies high in comparison with the fundamental field-line resonance frequency can also be generated in sub-resonators or waveguides formed inside the 
magnetosphere by steep gradients of Alfven velocity. The most essential gradient is related to the plasmapause. Also density irregularities in the plasmasphere can occur at different stages of plasmaspheric depleting and refilling (Singh and Horwitz, 1992). Another possibility is related to the formation of a waveguide for fast magnetosonic (FMS) waves in the F layer of the ionosphere (Klimushkin, 1998).

Experimental studies show that Pc2s are typical on the ground surface at high $\left(\Phi>65^{\circ}\right)$ geomagnetic latitudes and are attributed to cusp and auroral regions (Bering III et al., 1998; Engebretson et al., 2002). Bering III et al. (1998) mentioned $\approx 160 \mathrm{mHz}$ emissions in the geomagnetic field at South Pole station $\left(\Phi=-74^{\circ}\right)$. Engebretson et al. (2002) studied Pc1-2 events recorded simultaneously on the ground by the Antarctic meridional magnetometer chain located from the auroral $\left(\Phi=-66.5^{\circ}\right)$ to cusp $\left(\Phi=-74^{\circ}\right)$ geomagnetic latitudes, and in the outer magnetosphere by the POLAR satellite. The events described demonstrate common maxima on the ground and in space at $250-400 \mathrm{mHz}$.

In the present paper geomagnetic pulsations in the frequency range $70-200 \mathrm{mHz}(\mathrm{Pc} 2-3)$ are studied on the ground, in the $\mathrm{F}$ layer of the ionosphere, and in the magnetosphere.

In our earlier report (Yagova et al., 2009), we found that the Pc3 spectra in the F layer are enriched with high frequencies in comparison with ground observations. However, it was impossible to discriminate spatial from temporal variation on the basis of only CHAMP (CHAllenging Minisatellite Payload) satellite measurements. Moreover, spatial structures do exist in the ionospheric $\mathrm{F}$ layer and contribute to the ULF spectrum of magnetic field oscillations recorded by CHAMP. A detailed analysis of spatial structures near the plasmapause has been done by Heilig and Lühr (2013). Le et al. (2011) observed similar wavepackets at the low-orbiting ST-5 satellites. Their conclusion based on analysis of multisatellite and ground observations was that the apparent Pc2-3 waves were in fact the result of Doppler-shifted Pc4-5 waves.

In the present paper we analyze Pc2-3 waves at three locations: in the F layer of the ionosphere, on the ground, and in the magnetosphere, and our analysis shows that they cannot be completely explained by the Doppler effect. We give numerous examples of coherent $\mathrm{F}$ layer-ground and F layer-magnetosphere pulsations to show that the variations recorded by CHAMP at $f>70 \mathrm{mHz}$ are really Pc2-3 pulsations. The structure of the paper is as follows: observational data and data processing are described in Sect. 2, results are summarized in Sect. 3, calculations of Pc2-3 damping in the ionosphere are given in Sect. 3.4, and possible physical mechanisms are discussed in Sect. 4.

\section{Experimental data and data processing}

\subsection{Experimental data}

Pulsations with a central frequency above $70 \mathrm{mHz}$ are a rather typical phenomenon in the F layer. However, an ambiguity exists in the interpretation of pulsation measurements by low-orbiting satellites. The recorded variations of the geomagnetic field in pulsations' frequency range may be caused both by pulsations and satellite passage through quasi-periodic spatial structures in the ionosphere. In the general case of a finite $k_{\|}$(the projection of the wave vector to the satellite trajectory), the apparent frequency differs from the frequency at a stationary sensor due to the Doppler effect. To clarify the physical nature of Pc2-3s observed by CHAMP, we supplement the data recorded in the ionospheric F layer with data recorded on the ground surface and in the magnetosphere. The cross spectral analysis of data series measured simultaneously in the ionosphere and on the ground and/or by a high apogee satellite allows us to select pulsations coherent between the ionosphere and the ground or between the ionosphere and the magnetosphere. For such events, $k_{\|}$is low and the frequency observed by a fast-moving satellite should agree with one recorded by a stationary receiver on the ground surface or by a quasi-stationary receiver in the magnetosphere. We study pulsations measured simultaneously in the F layer-ground or F layer-magnetosphere locations, but not at magnetosphereground or magnetosphere-F layer-ground locations, because the occurrence rate of ground-magnetosphere $f>70 \mathrm{mHz}$ Pc2-3s is rather low due to the low amplitudes of ground Pc2$3 \mathrm{~s}$ and the relatively short time of THEMIS (Time History of Events and Macroscale Interactions during Substorms) satellite passes through the magnetospheric regions of high amplitudes and occurrence rates of Pc2-3s.

We use for the analysis data of 2 months of observations in 2007 (days 241-300) for which high-resolution data from the CHAMP and THEMIS-C satellites and the magnetometer network along the magnetic meridian 100 (MM100) are available. The statistical analysis is carried out for post-midnight (00:00-06:00) and afternoon-evening (14:00-20:00 MLT; magnetic local time) sectors. The geomagnetic conditions during the observation period are very quiet.

CHAMP was launched in 2000 into a quasi-circular orbit with $87^{\circ}$ inclination. The altitude during the operation was continuously decreasing and in 2007 it reached $350 \mathrm{~km}$. The geomagnetic field was measured at CHAMP with a three-component flux-gate magnetometer at a $50 \mathrm{~Hz}$ sampling rate. Detailed information about the mission can be found at http://isdc.gfz-potsdam.de. The European MM100 network of magnetometers (Heilig et al., 2007, 2010) ranges from auroral to middle latitudes $\left(42<\Phi<67^{\circ}\right)$ along approximately $\Lambda=100^{\circ}$. MM100 stations are equipped with three-component flux-gate magnetometers oriented along ge- 
Table 1. Coordinates of MM100 stations GOES projections.

\begin{tabular}{|c|c|c|c|c|c|c|}
\hline \multirow[t]{2}{*}{ Station } & \multicolumn{2}{|c|}{ Geographic } & \multicolumn{2}{|c|}{ CGM } & \multirow[t]{2}{*}{$L$} & \multirow{2}{*}{$\begin{array}{l}\text { UT of MLT } \\
\text { midnigh }\end{array}$} \\
\hline & Lat & Long & $\Phi$ & $\Lambda$ & & \\
\hline KIL & 69.02 & 20.79 & 65.94 & 103.73 & 6.11 & $21: 37$ \\
\hline SOD & 67.37 & 26.63 & 64.16 & 107.46 & 5.26 & $21: 12$ \\
\hline HAN & 62.30 & 26.65 & 59.01 & 104.68 & 3.77 & $21: 29$ \\
\hline NUR & 60.50 & 24.65 & 56.95 & 102.18 & 3.42 & $21: 45$ \\
\hline THY & 46.90 & 17.54 & 41.92 & 92.01 & 1.83 & $22: 12$ \\
\hline
\end{tabular}

ographic coordinates. The data rate for all the stations is $1 \mathrm{~s}$. The level of industrial interference differs from station to station. After preliminary data analysis, we selected five stations: THY, NUR, HAN, SOD, and KIL. For these stations Pc2-3 pulsations with peak-to-peak amplitudes above $0.1 \mathrm{nT}$ are clearly seen in the magnetograms. Their coordinates are given in Table 1. THEMIS magnetic measurements with $0.25 \mathrm{~s}$ resolution are available from February 2007. THEMIS-C crosses the magnetosphere within $\approx 12 \mathrm{~h}$ and the plasmasphere within $\approx 2 \mathrm{~h}$ during each orbit. The velocity of its ionospheric projection at middle latitudes is about $200 \mathrm{~m} \mathrm{~s}^{-1}$, i.e., it is low in comparison with CHAMP velocity, so THEMIS data can also be used to discriminate pulsations from spatial structures.

\subsection{Data processing}

Two horizontal components directed along corrected geomagnetic (CGM) coordinates ( $H$ and $D$ components) are used for the analysis of ground and CHAMP data. THEMIS data are rotated into a field aligned coordinate system with $\tau$ axis directed along the main magnetic field $\boldsymbol{B}, r$ is transverse to $\boldsymbol{B}$ and lies on the meridional plane (with inward positive direction) and $\varphi$ is chosen so that $r, t$, and $\varphi$ form a right-handed coordinate system.

During preliminary data analysis $128 \mathrm{~s}(\approx 2 \mathrm{~min})$ intervals with good data coverage were selected. In the frequency range studied, an artificial interference exists in CHAMP data formed by a series of triangle-like pulses repeated every $11 \mathrm{~s}$. This gives two narrow spectral peaks centered at about 90 and $170 \mathrm{mHz}$. For our case studies, we used the intervals free of this interference, and we also excluded the 80-105 and $160-180 \mathrm{mHz}$ frequency bands from the results of the automatic detection of Pc2-3s observed by CHAMP.

After high-pass filtering with the cutoff frequency $f_{L}=$ $20 \mathrm{mHz}$, we estimated the power spectral density (PSD), spectral coherence, and phase difference applying the Blackman-Tukey method (Chatfield, 2004). For the selected interval length $\left(n_{p}=128\right)$ and the window width $(m=32)$, the relative error of the spectral estimate $r=2 m / 3 n_{p}$ is $1 / 6$ (Kay, 1988) for the PSD spectra and it is $1 / 3$ for the spectral coherence and the phase difference.

Selection of Pc2-3 events is based on PSD spectrum analysis. Typically, a PSD in the Pc2-3 frequency range decreases with frequency as $P_{f}(f) \propto f^{-\alpha}$ (so-called color noise). We use a $\log -\log$ spectrum $\Pi(F)$, where $\Pi=\log \left(P_{f}\right)$ and $F=$ $\log (f)$. Note that for color noise, the dependence $\Pi_{\mathrm{cn}}(F)$, is linear $\Pi_{\mathrm{cn}}=a_{0}-a_{1} F$ ( $a_{0}$ and $a_{1}$ are constants). To stress the high frequency spectral maxima, $\Pi(F)$ is linearly detrended ("whitened") as $\Pi_{\mathrm{w}}(F)=\Pi(F)-\Pi_{\mathrm{cn}}(F)$ for each interval. In a $\Delta F$ vicinity of $F=F_{\max }$ where $\Pi_{\mathrm{w}}$ reaches a maximum, $\Pi_{\mathrm{w}}(F)$ is approximated by a square polynomial $\Pi_{\mathrm{w}}=-a_{2}\left(F-F_{\max }\right)^{2}+\Pi_{\mathrm{w} 0}$. An interval is selected as a Pc interval if both $\Pi\left(F_{\max }\right)$ and $a_{2}$ exceed some threshold values $\Pi_{b}$ and $a_{b}$. The Pc2-3 occurrence rate $\left(P_{\mathrm{Pc} 2-3}\right)$ is calculated as the ratio of total number of Pc2-3 intervals for a satellite(station) to the total number of intervals for which the PSD is calculated.

For the MM100 stations, the PSD threshold is determined by the background noise level and typical Pc2-3 amplitudes which are about $0.1 \mathrm{nT}$. The $a_{b}$ value is chosen after visual inspection of selected recordings under different values of threshold parameters as a compromise between the following two cases.

- high $a_{b}: P_{\mathrm{Pc} 2-3} \ll 1$ on the ground where the signal quality in the Pc2-3 frequency range is low;

- low $a_{b}: 1-P_{\mathrm{Pc} 2-3} \ll 1$ at CHAMP where Pc2-3s are typical.

As a result, the following set of selection parameters is taken:

$\Pi_{b}=-3.5, \quad a_{b}=f / 3$.

Here $f$ is in $\mathrm{mHz}$ and $\Delta F=0.15$.

The spectral coherence for all the possible combinations of the magnetic field components at each of the three locations (THEMIS, CHAMP, and the ground), as well as between the ionosphere-ground and the ionosphere-magnetosphere component pairs, was estimated to analyze the similarity of the signals at the different locations, and also the reliability of the phase difference and polarization estimates. The phase difference was analyzed at frequencies for which the spectral coherence $\gamma \geq 0.5$. Actually, for all the events selected as examples and discussed in Sect. 3.2, the maximal coherence for the CHAMP-ground and CHAMP-THEMIS-C component pairs exceeds 0.7 . This means that even if the $33 \%$ relative error of coherence estimates is taken into account $\gamma$ is high enough to allow us to analyze phase differences.

\section{Results of the analysis}

\subsection{Pc2-3s in the F layer, on the ground, and in the magnetosphere: main discrepancies}

The most important difference in geomagnetic pulsations at CHAMP compared to those on the ground surface is the higher amplitudes at frequencies $f>50 \mathrm{mHz}$. This effect 


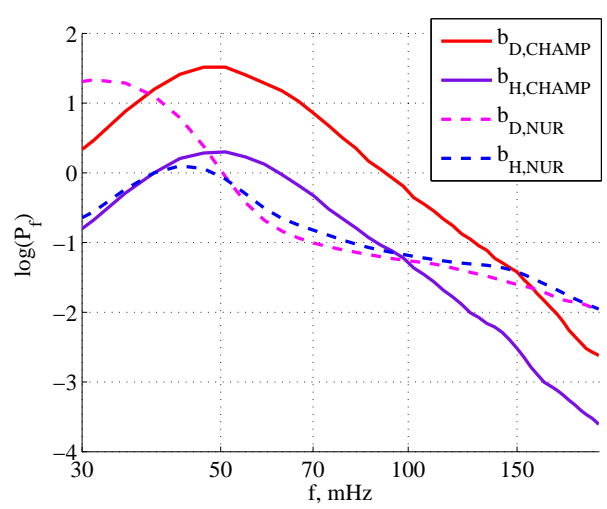

Figure 1. PSD spectra averaged over 6 days in 2007 (253-258) in the 12:00-18:00 MLT sector for CHAMP (solid lines) and NUR (dashed lines).

is illustrated in Fig. 1 in the PSD spectra averaged over 6 days of observations in September 2007 (DOY 253-258) at CHAMP and MM100 station NUR $(L=3.7)$. During this interval, CHAMP orbit was in the afternoon (12:00-16:00) and post-midnight (00:00-04:00 MLT) sectors. The results for the afternoon sector are shown in the figure. During the night the picture is qualitatively similar. The maximal PSD at CHAMP is recorded in the azimuthal $(D)$ component. In the frequency band $40-150 \mathrm{mHz}$, the $D$ component PSD at CHAMP exceeds the PSDs of both $H$ and $D$ components on the ground. The maximum of CHAMP $D$ to NUR $H$ PSD ratio is about an order of magnitude. The amplitudes of the vertical $(Z)$ component in the $\mathrm{F}$ layer is low in comparison with that for horizontal components, and so we analyze only horizontal components both in the ionosphere and on the ground.

\subsection{Examples of Pc2-3 in the F layer, on the ground, and in the magnetosphere}

Below we give three examples of coherent Pc2-3 ( $f>$ $70 \mathrm{mHz}$ ) pulsations recorded in the topside ionosphere by CHAMP together with signals simultaneously recorded on the ground or in the magnetosphere by THEMIS-C. The events were initially selected by an automated program developed for the detection of coherent Pc2-3s; then the signal waveforms were analyzed visually. All the events are presented in the same format: a figure with the signal time series, and another figure with the corresponding PSD spectra, spectral coherence, and phase difference. The data shown in time Figs. 2, 4, and 6 are high-pass filtered with a cutoff frequency $f_{L}=20 \mathrm{mHz}$. All the intervals in this section have the same $128 \mathrm{~s}$ duration, interval starts are given in UT, and dates are given in the YYYYDDD format, where YYYY is a year and DDD is a day number from the beginning of the year.

The events considered show that at least in some cases the Pc2-3s $(f>70 \mathrm{mHz})$ observed in the topside ionosphere

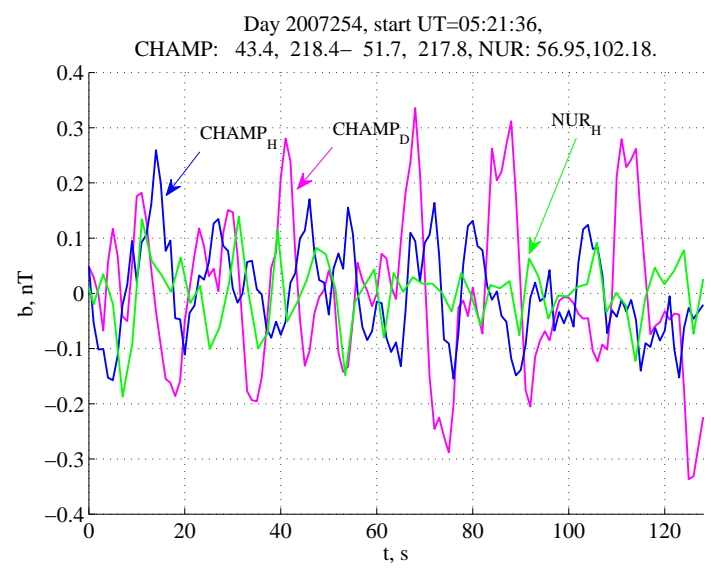

Figure 2. High-pass filtered $\left(f_{L}=20 \mathrm{mHz}\right)$ magnetograms for the CHAMP horizontal $\left(b_{H}\right.$ and $\left.b_{D}\right)$ components and the ground $b_{H}$ registered at the NUR station for the $128 \mathrm{~s}$ interval starting at 05:21:36 UT on day 2007254. CHAMP latitude and longitude values in CGM coordinates at the start and end of the interval shown and NUR CGM coordinates are given in the figure title.

were seen simultaneously with the same central frequency by an independent quasi-stationary sensor.

\subsubsection{A Pc2-3 event recorded simultaneously in the $F$ layer and on the ground starting at 05:21:36 UT on day 2007254}

An example of pulsations with a central frequency $f_{0} \approx$ $110 \mathrm{mHz}$ recorded by CHAMP and at the NUR station during a $128 \mathrm{~s}$ interval starting at 05:21:36 UT on day 2007254 UT is shown in Figs. 2 and 3. During this interval, CHAMP passed from CGM latitude $\Phi \approx 43$ to $52^{\circ} \mathrm{CGM}$ along the MM218 (magnetic meridian along $218^{\circ} \mathrm{CGM}$ longitude) in the afternoon sector (MLT $\approx 14: 00$ ). NUR is located at MM100 at the CGM latitude $\Phi \approx 57$ ( $L=3.42)$. During this interval, MLT $=08: 00$ at NUR, i.e., the station and the satellite were separated by $6 \mathrm{~h}$ in MLT.

Magnetograms for two horizontal components at CHAMP and the $H$ component at NUR are shown in Fig. 2. Peak-topeak amplitude reaches $0.5 / 0.3 \mathrm{nT}$ for the $D / H$ component in the F layer and $0.2 \mathrm{nT}$ on the ground surface. The amplitude of the $D$ component on the ground is below $0.1 \mathrm{nT}$ (not shown). The visible period is about $9 \mathrm{~s}$. It is more clearly seen in the CHAMP $D$ component and in the ground $H$ component.

The PSD spectra, spectral coherence, and phase difference for this interval are given in Fig. 3. A spectral maximum at $f_{0}=112 \mathrm{mHz}$ is clearly seen in the PSD spectrum of the CHAMP $D$ component. The $H$ component spectrum has no maximum at $f_{0}$ but there is a plateau near $f_{0}$ and a wide maximum in the spectral coherence between the two CHAMP horizontal components at $95<f<107 \mathrm{mHz}$, i.e., it is shifted to lower frequencies from $f_{0}$. The maxi- 


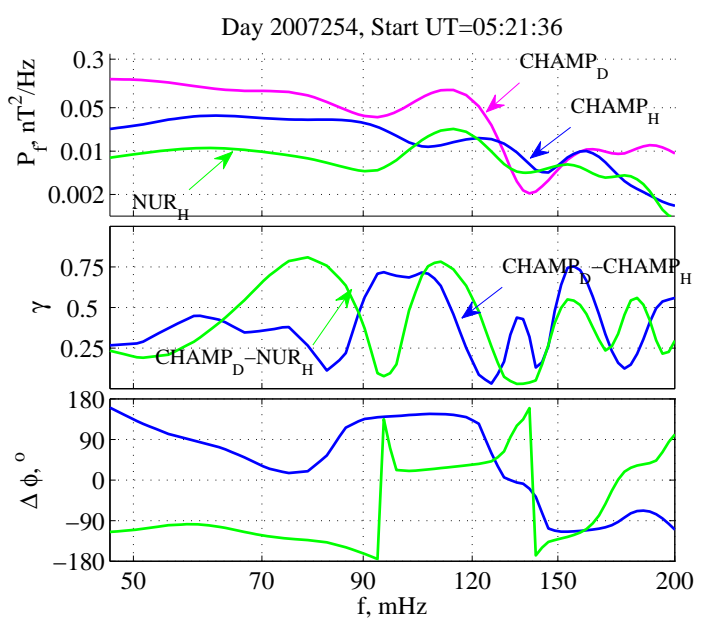

Figure 3. PSD spectra, spectral coherence, $\gamma$, and phase difference, $\Delta \varphi$, for the interval shown in Fig. 2.

mal spectral coherence between the two CHAMP horizontal components exceeds 0.75 , and the phase difference between the components at the frequency of maximal coherence $\Delta \varphi=140^{\circ}$, i.e., the polarization, is elliptical. The most interesting observation is the existence of a spectral maximum at NUR at the same frequency as at CHAMP (green curve in the upper panel of Fig. 3).

The spectral coherence also has a maximum near $f_{0}$, where $\gamma\left(f_{0}\right)=0.77$ (green curve in the middle panel of Fig. 3). The other maximum in spectral coherence with nearly the same value of $\gamma$ is seen at $f_{1} \approx 80 \mathrm{mHz}$. The phase differences in these two bands of high coherence are $-130^{\circ}$ and $25^{\circ}$, respectively (green curve in the bottom panel of Fig. 3).

So we see coherent pulsations with the central frequency $f_{0}=110 \mathrm{mHz}$ in the $\mathrm{F}$ layer in the afternoon (MLT) sector and on the ground surface in the morning sector. The PSD is higher in the azimuthal component in the F layer and in the meridional component on the ground surface. These components also demonstrate the maximal coherence among all the satellite-ground component combinations. The wave polarization in the ionosphere and on the ground surface corresponds to the shear Alfven mode.

\subsubsection{An example of a Pc2-3 event recorded simultaneously in the $F$ layer and in the outer magnetosphere starting at 08:03:44 UT on day 2007257}

Only a small fraction of CHAMP Pc2-3 pulsations can be recorded on the ground surface. That is why some other quasi-stationary reference observations are needed so that spatial and temporal variations can be distinguished. The other possibility to discriminate temporal and spatial variations is to compare the variations of the magnetic field measured simultaneously in the ionosphere and in the magne-

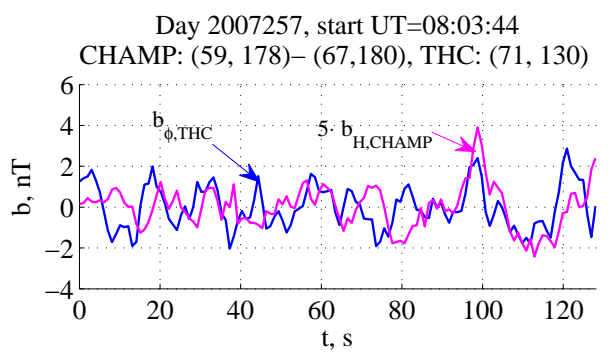

Figure 4. Filtered magnetograms for THEMIS-C $\left(b_{\varphi}\right)$ and CHAMP $\left(b_{D}\right)$ components for the $128 \mathrm{~s}$ interval starting at 08:03:44 UT on day 2007257. Average latitude and longitude values in CGM coordinates of the northern magnetic field line footprint of THEMIS-C's location during the interval shown are given in the figure title.

tosphere. For the pulsations in the frequency range studied (contrary to spatial variations or Pc4-5 pulsations recorded in the ionosphere as Pc2-3 due to the Doppler effect) the spectral coherence between the magnetosphere and the ionosphere should be high. Below we give some examples of such oscillations.

Pc2-3 pulsations are recorded simultaneously by the CHAMP and THEMIS-C satellites during a $128 \mathrm{~s}$ interval starting at 08:03:44 UT on day 2007257. CHAMP and THEMIS-C orbits are shifted from each other by about $2.5 \mathrm{~h}$ in MLT; CHAMP passes from $\Phi=59$ to $67^{\circ}$. THEMIS-C is located at $L=10$, at $-7^{\circ}$ from the equatorial plane; the CGM latitude of THEMIS-C ionospheric projection is $71^{\circ}$. Both satellites are in the near-noon sector (MLT $\approx 15: 00$ for CHAMP and 11:00 for THEMIS-C).

Magnetograms of the CHAMP $D$ component and the THEMIS-C azimuthal $(\varphi)$ component are shown in Fig. 4. The peak-to-peak amplitude is about $0.4 \mathrm{nT}$ at CHAMP and $2 \mathrm{nT}$ at THEMIS-C. Two apparent periods at about 12 and $7 \mathrm{~s}$ are clearly seen both in the CHAMP and THEMIS-C magnetograms.

The PSD spectra of all the THEMIS-C components and the CHAMP horizontal components are shown in Fig. 5. Two spectral maxima at $f=85$ and $130 \mathrm{mHz}$ are seen in the PSD spectra of both the CHAMP and the THEMIS$\mathrm{C}$ azimuthal components (solid lines in the upper panel of Fig. 5). The maximum at $130 \mathrm{mHz}$ is seen also in the PSD spectra of the THEMIS-C radial component and the CHAMP $H$ component (dashed lines in the upper panel of Fig. 5) and in the THEMIS-C longitudinal component (red dash-dot line in the upper panel of Fig. 5). At $f=85 \mathrm{mHz}$ the azimuthal component PSDs (the THEMISC $P_{f, \varphi}$ and the CHAMP $\left.P_{f, D}\right)$ are higher than the radial/meridional PSDs for both satellites. The THEMIS-C longitudinal PSD is comparable with the azimuthal one. At $f=130 \mathrm{mHz}$, the spectral ratio $P_{f, \tau} / P_{f, \varphi}$ reaches 2.5 and the THEMIS-C radial and azimuthal PSDs are approximately equal. The CHAMP meridional-to-azimuthal spectral ratio $P_{f, H} / P_{f, D} \approx 3$. Thus, both the CHAMP and the THEMIS- 
C wave polarizations correspond to the sum of the compressional and the shear Alfven modes with a higher contribution of the compressional mode at the higher frequency maximum.

The spectral coherence is shown in the middle panel of Fig. 5 for the all three CHAMP D-THEMIS-C component combinations. Both spectral maxima are clearly seen in the spectral coherence with $\gamma>0.5$ for both THEMIS$\mathrm{C}$ transverse components (blue and green lines in the middle panel of Fig. 5). For the longitudinal THEMIS-C component, only the maximum at the higher frequency $f=130 \mathrm{mHz}$ is seen in the spectral coherence. The spectral coherence for the THEMIS-C-CHAMP $H$ component pairs has maxima at nearly the same frequencies as for the THEMIS-CCHAMP $D$ component pairs but $\gamma$ is somewhat lower: the inter-component spectral coherence for each satellite both at 85 and $130 \mathrm{mHz}$ exceeds 0.5 (not shown).

Phase differences are shown in the bottom panel of Fig. 5 . The signal polarization also differs at the frequencies of two spectral maxima. The THEMIS-C azimuthal and radial components are shifted by about $\pi / 2$ both at 85 and $130 \mathrm{mHz}$ (green line in the bottom panel of Fig. 5). The phase difference between the THEMIS-C azimuthal and longitudinal components changes from 0 at $85 \mathrm{mHz}$ to $-\pi / 2$ at $130 \mathrm{mHz}$ (red line in the bottom panel of Fig. 5), and the phase difference between the longitudinal and the radial components is $-\pi / 2$ at $85 \mathrm{mHz}$ and $\pi$ at $130 \mathrm{mHz}$ (not shown). The CHAMP $D$ component is shifted by $\pi / 2$ at $85 \mathrm{mHz}$ from the azimuthal THEMIS-C component (magenta line in the bottom panel of Fig. 5) and they are in phase at $f=130 \mathrm{mHz}$. The phase difference between the CHAMP $H$ and $D$ components is approximately the same as between the THEMIS-C azimuthal and longitudinal components. It changes from 0 at $85 \mathrm{mHz}$ to $-\pi / 2$ at $130 \mathrm{mHz}$ (cyan line in the bottom panel of Fig. 5).

Taking into account that the compressional mode contributes mostly to the CHAMP $H$ component and the shear Alfven wave dominates in the $D$ component in the $\mathrm{F}$ layer and in the azimuthal component in the magnetosphere, we can speculate that the observed correspondence in the polarizations between the magnetospheric and the ionospheric signals, along with the similar PSD spectra and the high spectral coherence indicate that the signals in these two locations are of the same origin. The phase shift between the pulsations in the magnetosphere and in the F layer may be caused by wave propagation.

\subsubsection{An example of a Pc2-3 event recorded simultaneously in the $F$ layer and in the plasmasphere starting at 01:38:40 UT on day 2007286}

The next example presents a Pc2-3 pulsation recorded simultaneously by CHAMP in the F layer and by THEMIS$\mathrm{C}$ inside the plasmasphere during a $128 \mathrm{~s}$ interval start-

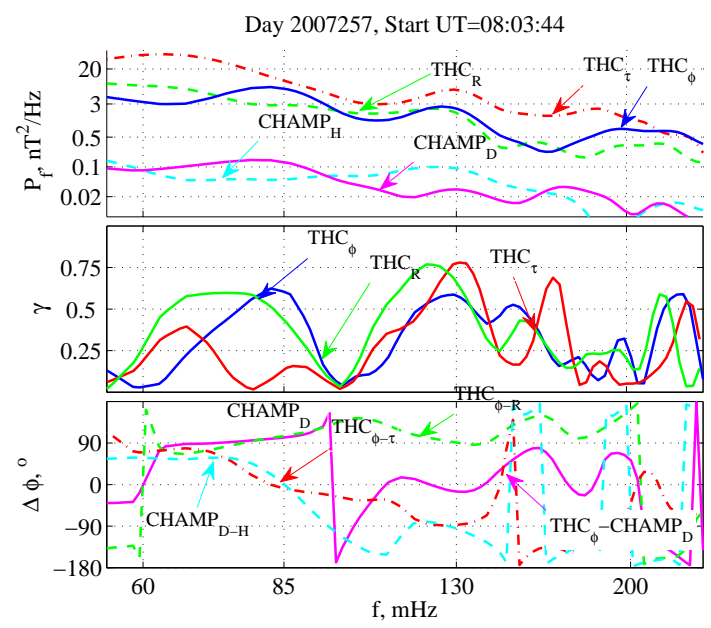

Figure 5. PSD spectra, spectral coherence, $\gamma$, and phase difference, $\Delta \varphi$, for the three THEMIS-C and the two horizontal CHAMP components for the interval shown in Fig. 4.

ing at 01:38:40 UT on day 2007286. During this interval, THEMIS-C was near the plasmapause position $(L=4.3)$ and CHAMP was passing from $\Phi=-35$ to $-27^{\circ}$ along MM230. Both satellites were in the near-noon sector (MLT $\approx 12: 00$ for CHAMP and $\approx 10: 00$ for THEMIS).

Magnetograms of the THEMIS-C azimuthal component and the CHAMP $D$ component are shown in Fig. 6. Pulsations with $90 \mathrm{mHz}$ central frequency and peak-to-peak amplitude $0.3-0.5 \mathrm{nT}$ were seen by both satellites on the background of $\approx 25 \mathrm{mHz} \mathrm{Pc} 4$ pulsations with the peak-to-peak amplitude of $\approx 1 \mathrm{nT}$.

The PSD spectra for this event are given in the upper panel of Fig. 7 for three THEMIS-C components (green, blue, and red lines) and the CHAMP horizontal components (cyan and magenta lines). Two maxima are seen at about 90 and $130 \mathrm{mHz}$. At $f=90 \mathrm{mHz}$, the THEMIS-C radial PSD is at about 2-3 times higher than the azimuthal and the longitudinal PSD. At $f=130 \mathrm{mHz}$ PSDs of all three THEMIS-C components are similar. The CHAMPto-THEMIS-C spectral ratio $P_{f, \mathrm{CHAMP}, D} / P_{f, \mathrm{THC}, \varphi} \approx 7$ for the azimuthal components and $P_{f, \text { CHAMP, } H} / P_{f, \text { THC, } R} \approx 2$ at $f=90 \mathrm{mHz}$. At $130 \mathrm{mHz} P_{f, \text { CHAMP }, D} / P_{f, \text { THC }, \varphi} \approx 3$ and $P_{f, \text { CHAMP }, H} / P_{f, \text { THC }, \varphi} \approx 1$. Thus, for this event the wave amplitude in the $\mathrm{F}$ layer is significantly higher than in the magnetosphere. The CHAMP-to-THEMIS PSD ratio at $f=$ $90 \mathrm{mHz}$ is higher in the azimuthal component corresponding to the shear Alfven mode at $f=90 \mathrm{mHz}$. During this interval, a Pc2-3 pulsation occurs simultaneously with a Pc4 pulsation with a period of about $40 \mathrm{~s}(25 \mathrm{mHz})$. Pc4 amplitudes are the same in the ionosphere and in the magnetosphere (Fig. 6).

Spectral coherence for all the CHAMP D-THEMIS-C component pairs is shown in the middle panel of Fig. 7. Both 90 and $130 \mathrm{mHz}$ spectral maxima are also seen in the 


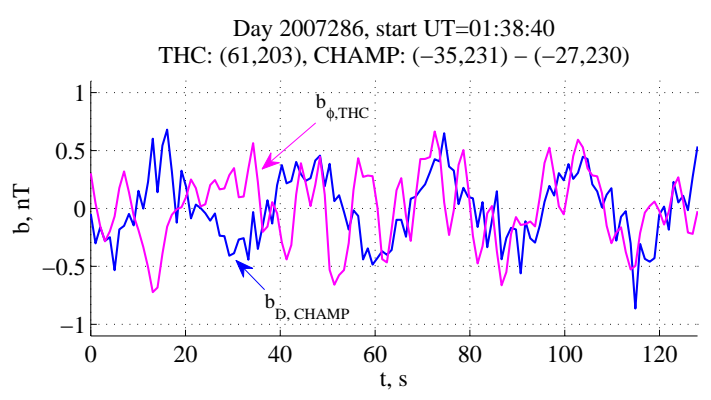

Figure 6. Filtered magnetograms for THEMIS-C $\left(b_{\varphi}\right)$ and CHAMP $\left(b_{D}\right)$ components for the $128 \mathrm{~s}$ interval starting at 01:38:40 UT on day 2007286. Average latitude and longitude values in CGM coordinates of the northern magnetic field line footprint of THEMIS-C's location during the interval shown are given in the figure title.

spectral coherence. The higher frequency maximum is seen in all three THEMIS-C components, and for all three components, the maximal values of coherence reach or exceed 0.5 . The coherence maximum at $f=90 \mathrm{mHz}$ can be seen only in the transverse components (green and blue curves in the middle panel of Fig. 7). The spectral coherence between the CHAMP $D$ component and the THEMIS-C longitudinal component has a maximum at $f=70 \mathrm{mHz}$. The maxima of the CHAMP $H$ component spectral coherence are at nearly the same frequencies but maximal coherence is lower than for the CHAMP $D$ component (not shown).

The phase differences for this event are shown in the bottom panel of Fig. 7. The phase difference between the azimuthal and the radial (green) and the azimuthal and the longitudinal (red) THEMIS-C components is about $\pi / 4$ at $f=90 \mathrm{mHz}$, and thus the radial and the longitudinal pulsations are in phase at this frequency. At $f=130 \mathrm{mHz}$ $\Delta \varphi_{\mathrm{THC}, \varphi-\mathrm{THC}, R}$ is about zero and $\Delta \varphi_{\mathrm{THC}, \varphi-\mathrm{THC}, \tau}$ is almost $\pi$, i.e., the pulsation polarization in the magnetosphere is elliptical at $f=90 \mathrm{mHz}$ and almost linear at $f=130 \mathrm{mHz}$. The phase difference between the CHAMP horizontal components (cyan) changes from $3 / 4 \pi$ at $f=90 \mathrm{mHz}$ to $-\pi / 2$ at $f=130 \mathrm{mHz}$, and thus the difference in polarization is almost $\pi$ like for the THEMIS-C azimuthal-longitudinal component pair. The phase difference between the THEMIS$\mathrm{C}$ azimuthal component and the CHAMP $D$ component $\Delta \varphi_{\mathrm{THC}, \varphi-\mathrm{CHAMP}, D}$ is about $\pi$ at $f=90 \mathrm{mHz}$ and it is almost zero at $f=130 \mathrm{mHz}$. The phase difference between the THEMIS-C longitudinal and the CHAMP $H$ component $\Delta \varphi_{\text {THC, } \tau-\text { CHAMP, } H}$ (not shown) changes from $-\pi / 2$ at $f=90 \mathrm{mHz}$ to $\pi / 2$ at $f=130 \mathrm{mHz}$, i.e., the corresponding components in the two locations demonstrate similar phase dependence on frequency.

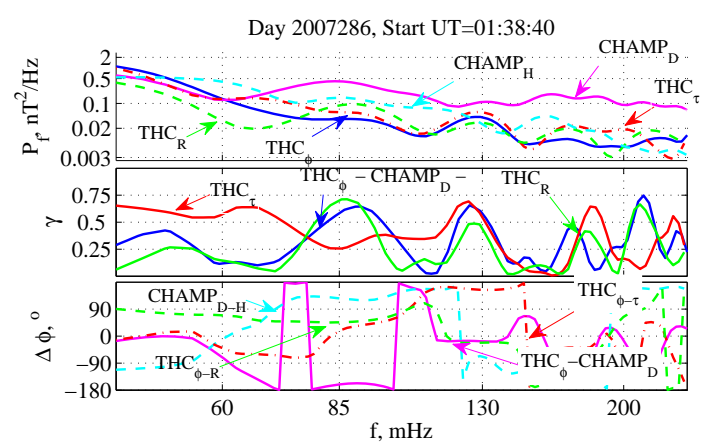

Figure 7. PSD spectra, spectral coherence, $\gamma$, and phase difference, $\Delta \varphi$, for the three THEMIS-C and the two horizontal CHAMP components for the interval shown in Fig. 6.

\subsection{Pc2-3 in the F layer, in the magnetosphere, and on the ground surface: spatial distribution}

In this subsection some statistical results for the pulsations shown as examples in the previous subsection are presented. The latitude distribution of the Pc2-3 $(70-140 \mathrm{mHz})$ occurrence rate $\left(P_{\mathrm{Pc} 2-3}\right)$ and that of the PSD are given in Fig. 8 for pulsations recorded by CHAMP in two MLT sectors over 2 months in 2007 (days 241-300). The Pc2-3 occurrence rate is higher for the CHAMP $D$ component in both the afternoon (left panels) and the post-midnight (right panels) sectors. The occurrence rate during the night has a clear maximum at $\Phi \pm 57^{\circ} \mathrm{CGM}$ and a second one at low latitudes at $|\Phi|<30^{\circ}$ CGM. During the day the picture differs for the two hemispheres.

In the Southern Hemisphere $P_{\mathrm{Pc} 2-3}$ for the $D$ component grows from low to high latitudes and in the Northern Hemisphere the maximum is expressed weakly. $P_{\mathrm{Pc} 2-3}$ in the meridional component during the day has a low-latitude maximum at $\Phi \pm 30^{\circ}$, but during the night, pulsations in the $H$ component almost disappear. The PSD grows towards high latitudes, and during the day it is higher in the azimuthal than in the meridional component. During the night, the difference in PSD between components is not significant. The highest occurrence rates and amplitudes are seen at night at about $\Phi= \pm 57^{\circ}$ and in the afternoon at $\Phi>60^{\circ}$.

Pc2-3 at $f>70 \mathrm{mHz}$ are regularly seen on the ground surface at night in the $H$ component. The latitude distribution of the Pc2-3 occurrence rate and the PSD for the $H$ component pulsations recorded along the MM100 magnetometer array in the post-midnight (MLT) sector $(00: 00<$ MLT $<06: 00)$ are presented in Fig. 9. Under the same set of selection parameters as for CHAMP (1), the occurrence rate is an order of magnitude lower, and the PSD is 3-5 times lower on the ground in comparison with the $\mathrm{F}$ layer. The main maximum corresponds to $L=5\left(\Phi=64^{\circ}\right.$, SOD) and a secondary maximum is seen at $L=3.4\left(\Phi=57^{\circ}, \mathrm{NUR}\right)$. At THY $\left(\Phi=47^{\circ}\right.$, $L=1.8$ ), Pc2-3 disappears almost completely. 

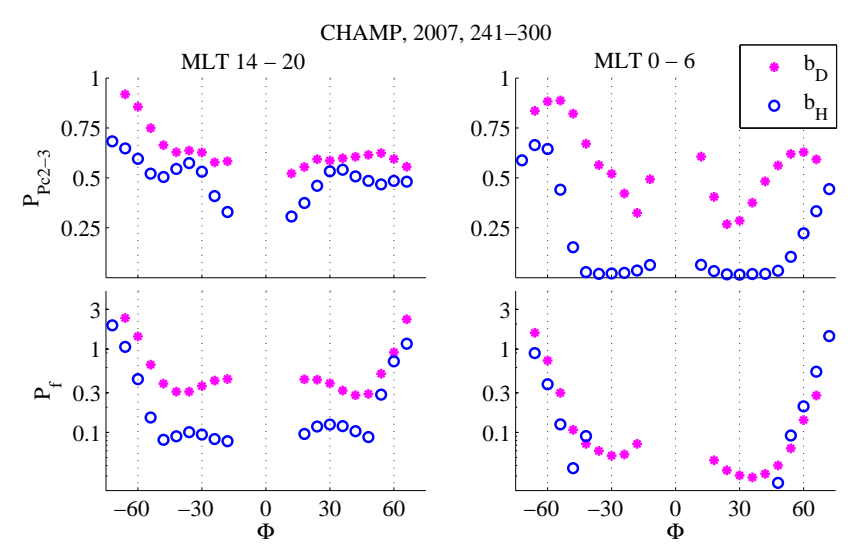

Figure 8. Latitude distribution of the occurrence rate (upper panel)and PSD (bottom panel) for CHAMP Pc2-3 in two MLT sectors over 2 months in 2007. Two components are given by different markers and colors.

We analyze the spatial distribution of the magnetospheric Pc2-3s with the set of parameters (1) in the same MLT sectors as for CHAMP, using the THEMIS-C data in the MLT intervals (00:00-06:00) and (12:00-20:00) (the last interval is extended to provide a sufficient data array) and during the same days (241-300). During the days selected for the analysis THEMIS-C crossed $L$ shells from 1.5 to 7.5 in the afternoon (MLT) sector, while in the post-midnight sector it crossed all the $L$ shells outside $L=2$. The results for the THEMIS-C Pc2-3 occurrence rate and PSD are given in Fig. 10 in the same format as for CHAMP in Fig. 8. The maximum of the occurrence rate falls within $L$ values from 4 to 6 in both the afternoon and post-midnight sectors. In both sectors at high $L$, the occurrence rate and the PSD for the field-aligned $\left(b_{\tau}\right.$, green stars) component pulsations are nearly the same as for transverse components $\left(b_{R}, b_{\varphi}\right)$, while at low $L$ they are small in comparison with $b_{R}, b_{\varphi}$. The occurrence rate is higher in the afternoon sector. In the post-midnight sector, the Pc2-3 occurrence rate and PSD slowly decrease with $L$ for $L>6$ and Pc2-3 are recorded at least up to $L=10$.

Comparison of Pc2-3 parameters near the equatorial plane of the magnetosphere and in the F layer shows that in the post-midnight (MLT) sector, the maximum of the $\mathrm{F}$ layer occurrence rate is found at $L \approx 3.5\left(|\Phi| \approx 57^{\circ}\right)$ in the $\mathrm{F}$ layer and at $4<L<6$ in the magnetosphere. The Pc2-3 occurrence rate in the ionosphere is several times higher than in the magnetosphere. During the night, pulsation amplitudes are comparable in the ionosphere and the magnetosphere, and the maximal PSD of afternoon pulsations is 3-5 times higher in the F layer. The polarization is also different. In the F layer, pulsation spectral power and occurrence rate are higher in the azimuthal component, and in the magnetosphere all three components have comparable amplitudes at $L>4$. Inside the plasmasphere, amplitude and occurrence rate of $b_{\tau} \operatorname{Pc} 2-3$ are low in comparison with those for transverse components.

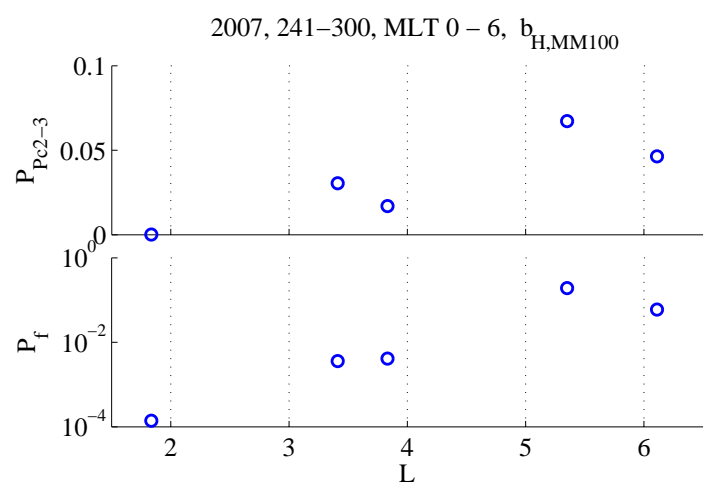

Figure 9. $L$ distribution of the occurrence rate (upper panel)and PSD (bottom panel) along MM100 in the 00:00-06:00 MLT sector over 2 months in 2007 for the meridional $\left(b_{H}\right)$ component.

The high-latitude boundary of the F layer Pc2-3 occurrences lies at about $L=4$ and may be related to the plasmapause. To check this assumption we compare the day-to-day variations of the $L$ value of the Pc2-3 occurrence rate maximum $\left(L_{\mathrm{Pc} 2-3}\right)$ with the plasmapause position determined from the Carpenter formula $L_{\mathrm{pp}}=5.7-0.47 \mathrm{Kp}$ (Carpenter and Anderson, 1992). For low and moderate Kp, the empirical formula obtained from CHAMP observations by Heilig and Lühr (2013) gives similar results. Two-day mean values of $L_{\mathrm{Pc} 2-3}$, determined from CHAMP measurements in the 00:00-06:00 MLT sector in the Southern Hemisphere during the best data coverage for 2 months, are shown in Fig. 11 together with $L_{\mathrm{pp}}$ estimated from 3-day mean Kp values. $L_{\mathrm{Pc} 2-3}(t)$ and $L_{\mathrm{pp}}(t)$ in Fig. 11 are determined as $\left\langle L_{\mathrm{Pc} 2-3}\right\rangle \mid \tau_{\mathrm{Pc} 2-3}$ and $\left\langle L_{\mathrm{pp}}\right\rangle \mid \tau_{\mathrm{pp}}$, respectively, where angle brackets indicate time averaging over an interval $\tau$, and where $\tau_{\mathrm{Pc} 2-3}=[-2,0]$ days, and $\tau_{\mathrm{pp}}=[-3,0]$ days. $L_{\mathrm{Pc} 2-3}$ varies within the range $2<L<4$.1, i.e., at about $L_{\mathrm{pp}}-2$ and the day-to-day variations of $L$ for the Pc2-3 occurrence rate maximum and plasmapause demonstrate positive correlation.

\subsection{Model calculations}

MHD wave transmission through and reflection from a thin ionosphere has been studied theoretically in numerous papers beginning with (Nishida, 1964; Hughes and Southwood, 1976). After this initial period, the problem has also been studied for more general geometries of the background magnetic field (see, e.g., Leonovich and Mazur, 1991; Alperovich and Fedorov, 1993; Sciffer and Waters, 2002; Sciffer et al., 2004, 2005). Below we present a model and numerical results for the problem of magnetohydrodynamic wave transmission through and reflection from the ionosphere within a more realistic "thick ionosphere" model (Knudsen et al., 1992). Our analysis follows the scheme developed by Budden (1966) and Alperovich and Fedorov (2007). It is shown in Sect. 3.3 that the Pc2-3 polarization corresponding to the shear Alfven mode dominates in the plasmasphere and in the 


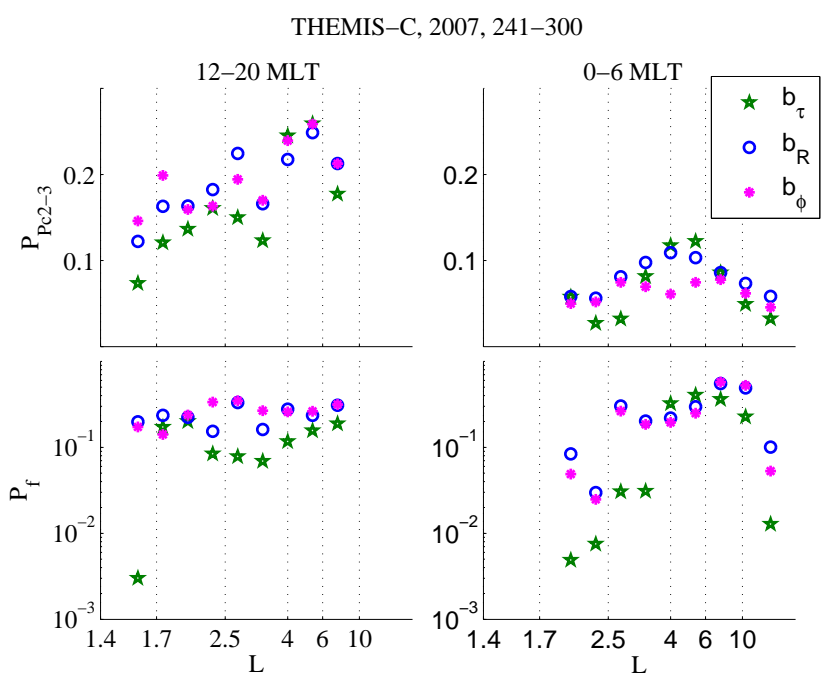

Figure 10. $L$ distribution of the occurrence rate (upper panel) and PSD (bottom panel) for THEMIS-C Pc2-3 in two MLT sectors over 2 months in 2007. Three components are given by different markers and colors.

mid-latitude ionosphere. In this section, we consider theoretically an incident shear Alfven wave transmission through and reflection from the ionosphere.

We assume a plane stratified model of the ionosphere: the dip-angle $I$ of the geomagnetic field $\boldsymbol{B}_{0}$ is constant, and the dielectric permeability tensor $\hat{\varepsilon}=\hat{\varepsilon}(z)$ depends only on height. We introduce an oblique coordinate system $\left\{x^{1}, x^{2}, x^{3}\right\}$ with a southward $x^{1}$ axis, and an eastward $x^{2}$ axis. The angle between $x^{3}$ and $x^{1}$ is equal to the dipangle $I$. The $x^{3}$ axis is parallel to the field-lines, and the coordinate surface $x^{3}=$ const is a horizontal plane; $x^{3}=0$ is at the Earth's surface, and $x^{3}=z$ is a horizontal plane at a height $z$ above the Earth's surface. We consider the case of waves propagating in the meridional plane. For the harmonics $\propto \exp \left(-i \omega t+i k_{1} x^{1}\right)$, the horizontal components of the electric $E$ and magnetic $B$ fields can be written as

$$
\left[\begin{array}{l}
\boldsymbol{B}\left(x^{3}\right) \\
\boldsymbol{E}\left(x^{3}\right)
\end{array}\right] \exp \left(i k_{1} x^{1}-i \omega t\right)
$$

where $\omega$ is the angular frequency of the wave, $k_{1}$ is a wave vector component, and $t$ is time. Taking into account that the longitudinal component of the electric field $E_{3}$ is zero, from Maxwell's equations we get

$$
\begin{aligned}
& \partial_{3} B_{1}=-\mu_{0} \sigma_{2} E_{1} / \sin I+\mu_{0} \sigma_{1} E_{2}+i k_{1} B_{1} \cot I, \\
& \partial_{3} B_{2}=-\mu_{0} \sigma_{1} E_{1} / \sin ^{2} I-\mu_{0} \sigma_{2} E_{2} / \sin I \\
& \partial_{3} E_{1}=i \omega B_{2} \\
& \partial_{3} E_{2}=i k_{1} E_{2} \cot I-i \omega B_{1} .
\end{aligned}
$$

Here $k_{0}=\omega / c$ and $\sigma_{1,2}\left(x^{3}\right)=-i \omega \varepsilon_{1,2}\left(x^{3}\right)$ are the complex conductivities. In the $\mathrm{E}$ layer of the ionosphere $\sigma_{1,2}=$

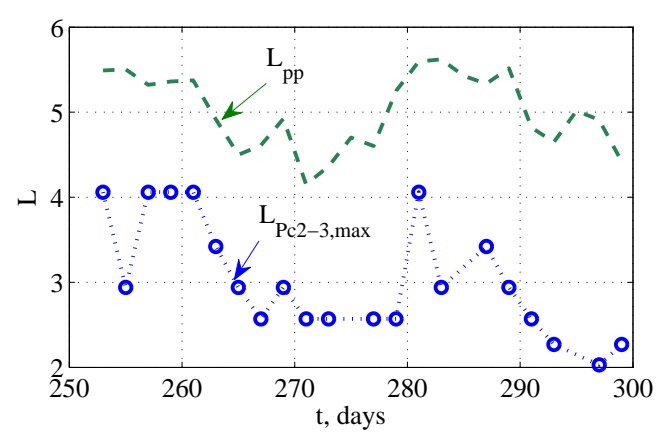

Figure 11. Day-to-day variations of the position of maximal Pc2-3 occurrence rate at CHAMP, in the 00:00-06:00 MLT sector for two months in 2007, and the plasmapause position, determined from the two-day mean Kp with Carpenter's formula.

$\sigma_{\mathrm{P}, \mathrm{H}}\left(x^{3}\right)$, i.e., the Pedersen and Hall conductivities, and in the $\mathrm{F}_{2}$ layer and further up $\sigma_{1}=-i \omega / \mu_{0} V_{A}^{2}$.

Let a shear Alfven wave with an amplitude $\boldsymbol{B}^{(i)}, \boldsymbol{E}^{(i)}$ at $x_{3}=z_{1}$ be incident on the ionosphere. The total field at $x_{3}=z_{1}$ can be represented as $\boldsymbol{B}^{(i)}+\boldsymbol{B}^{(r)}$, where $\boldsymbol{B}^{(i)}$ is the magnetic component of the incident wave and $\boldsymbol{B}^{(r)}$ is the reflected field. The matrix for the reflected and incident waves is $\boldsymbol{B}^{(r)}=\boldsymbol{R} \boldsymbol{B}^{(i)}$, where $\boldsymbol{R}$ is the reflection coefficient matrix. To find the wave amplitudes on the ground surface, first the fields reflected from the ionosphere are found and then, from the known values of the initial incident field and the reflected field, the full field at some reference level $z_{1}$ is calculated. Then the ground electric and magnetic field are found from the electric and magnetic field at $z_{1}$.

Reflection coefficients at $z_{1}$ are calculated via the admittance $\boldsymbol{Y}$ or impedance $\boldsymbol{Z}=\boldsymbol{Y}^{-1}$ matrices, which relate horizontal components of the electric $\boldsymbol{E}_{\tau}$ and magnetic $\boldsymbol{B}_{\tau}$ fields, $\boldsymbol{B}_{\tau}=\boldsymbol{Y} \boldsymbol{E}_{\tau}$, and $\boldsymbol{E}_{\tau}=\boldsymbol{Z} \boldsymbol{B}_{\tau}$. By substituting $\boldsymbol{B}_{\tau}=\boldsymbol{Y} \boldsymbol{E}_{\tau}$ and $\boldsymbol{E}_{\tau}=\boldsymbol{Z} \boldsymbol{B}_{\tau}$ into Maxwell equations, the Ricatti-type matrix equations are obtained for the admittance and impedance matrices (see, e.g., Budden, 1966).

If the Wait-Price condition (the strong skin effect approximation, $k \delta_{g} \ll 1$, where $\delta_{g}=\left(2 / \omega \mu_{0} \sigma_{g}\right)^{1 / 2}$ is the skin depth) is valid, the impedance conditions at the ground surface are $E_{1}=-Z_{g} B_{2}, E_{2}=Z_{g} B_{1}$. A surface impedance $Z_{g}=Z_{g}(\omega)$ is determined by the Earth conductivity distribution $\sigma_{g}\left(x^{3}\right)$ and within the strong skin effect approximation $Z_{g}$ does not depend on the wave's spatial structure. For a homogeneous half-space under strong skin effect $Z_{g}=\exp (-i \pi / 4) \sqrt{\omega \mu_{0} / \sigma_{g}}$. Then the admittance $\boldsymbol{Y}\left(x^{3}\right)$ and $\boldsymbol{Z}\left(x^{3}\right)$ matrices are recalculated step-by-step through the atmosphere and the ionosphere with Ricatti-type matrix equations from the ground surface to the altitude $z_{1}$ (we set $\left.z_{1}=2000 \mathrm{~km}\right)$. The matrix $\boldsymbol{Y}\left(z_{1}\right)$ is used to calculate the reflection matrix $\boldsymbol{R}$ and the total field at the altitude $z_{1}$.

The transmission coefficient depends on wave frequency and wave number. Pulsations with a spatial scale less than the Earth-ionosphere distance are attenuated as $\exp (-k h)$ 


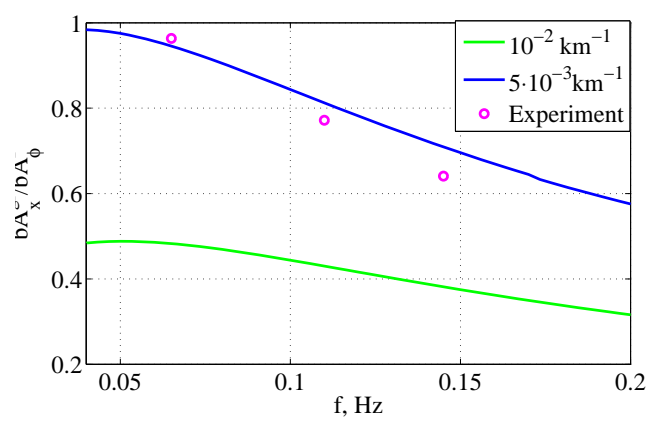

Figure 12. Ground-to-ionosphere amplitude ratio for Alfven waves obtained from the full wave equation with International Reference Ionosphere (IRI) model ionosphere at two different $\boldsymbol{k}$ values (solid lines) and the NUR to CHAMP amplitude ratio, averaged over two months, in three frequency bands (magenta circles)

by the ionosphere (Hughes and Southwood, 1976). We have calculated the spectrum of the ground-to-ionosphere amplitude ratio for $2 \times 10^{-3}<k<10^{-2} \mathrm{~km}^{-1}$. The results are shown in Fig. 12 for $350 \mathrm{~km}$ altitude. The IRI ionosphere model (Bilitza, 2001) is used with the following parameters: day 2007253 (12 September), MLT $=02: 00$, CGM latitude $\Phi=57^{\circ}$, and CGM longitude $\Lambda=102^{\circ}$. The groundto-ionosphere amplitude ratio decreases with frequency and wave number. For comparison, we used the ground-toionosphere amplitude ratio of Pc2-3s recorded at NUR in the $H$ component and by CHAMP in the $D$ component. Pc2-3 events are automatically selected for all CHAMP orbit segments at CGM latitudes $-60<\Phi<-35^{\circ}$ in three frequency bands $(50-80,105-130$, and $130-160 \mathrm{mHz})$ in the post-midnight (MLT) sector (00:00-06:00) for two months of observations: 2007 , days $241-300$. The best agreement between calculated and measured data is found for $k=5 \times$ $10^{-3} \mathrm{~km}^{-1}$. The maximal Doppler shift corresponds to the case $\boldsymbol{k}=k_{\|}$. For the $k=5 \times 10^{-3} \mathrm{~km}^{-1}$ waves recorded by a spacecraft moving at a $8 \mathrm{~km} \mathrm{~s}^{-1}$ speed, $\Delta \omega=k v=4 \times$ $10^{-2} \mathrm{~s}^{-1}$, i.e., $\Delta f=6.4 \mathrm{mHz}$. Thus, the observed groundto-ionosphere amplitude ratio corresponds to a wave spatial scale which could provide a Doppler shift within a few percent of the apparent frequency of the Pc2-3 pulsations as recorded by a low-orbiting spacecraft.

\section{Discussion and conclusion}

Our analysis of individual Pc2-3 cases and statistical properties of these pulsations have shown that pulsations with frequencies exceeding the fundamental Alfven resonance frequency are typical in the ionospheric F layer and in the magnetosphere. The analysis of simultaneous ground and ionospheric Pc2-3 observations in the post-midnight and afternoon (MLT) sectors shows that ground Pc2-3s are regularly observed during the night and are almost absent at the afternoon. For Pc2-3s registered simultaneously in the F layer and on the ground, the amplitudes are several times lower on the ground than in the ionosphere. The existence of coherent pulsations recorded by the fast-moving CHAMP satellite in the $\mathrm{F}$ layer and by ground magnetometers or by the high apogee THEMIS-C satellite shows that the F layer Pc2-3 cannot be interpreted either as a result of spatial sampling of structured inhomogeneities by the fast-moving spacecraft, or as Doppler shifted Pc4-5s. However, this result of the present study is only qualitative. Currently we cannot estimate the contribution of the Pc2-3s and the Doppler shifted Pc4-5s with apparent Pc2-3 frequencies to the observed F layer pulsations. The problem is that a non-zero fraction of highly coherent intervals exists for any two time series. Therefore, for case studies, signal waveforms should be analyzed along with PSD and coherence spectra to discriminate between coherent events of common origin and those resulting from a random coincidence.

Moreover, the simultaneous activation of pulsations in the Pc4-5 and Pc2-3 frequency ranges in the magnetosphere is possible (Balasis et al., 2012). The ground-to-ionosphere amplitude ratio is such a case determined by the dependence of transmission and reflection coefficients on wave frequencies and wavelengths and different combinations of Pc2-3 and Pc4-5 in two locations can be observed. For example, if Pc23 amplitudes in the $\mathrm{F}$ layer are within several tenths of $\mathrm{nT}$ and wavelengths $k \geq 10^{-2} \mathrm{~km}^{-1}$, only Pc2-3 would be recorded by an ionospheric satellite due its high speed, and only Pc4-5 would be seen on the ground surface because of the effective ionospheric attenuation of Pc2-3s. This also means that synchronous recording of a ground Pc5 and an ionospheric Pc23 does not necessarily indicate that the ionospheric signal is a Doppler shifted Pc4-5. Thus, further case studies of Pc2-3s recorded simultaneously in several locations are necessary. Also a technique for the automatic detection of coherent pulsations which takes into account the Doppler effect should be developed.

Several physical mechanisms may be responsible for the generation of magnetohydrodynamic waves in the 70$200 \mathrm{mHz}$ frequency range in the magnetosphere. The first one is related to the ion cyclotron resonance. Pc2-3 frequencies are close to the oxygen cyclotron frequencies in the outer magnetosphere. The distribution of particle and plasma parameters near the plasmapause is rather complicated due to the combination of steep plasma gradients at the plasmapause and the oxygen torus (Nose et al., 2011). This may lead to different dependencies of the Pc1-2 frequency on the concentration of heavy ions (Klimushkin et al., 2006; Mikhailova, 2014). However, $\mathrm{O}^{+}$ions are observed in the outer magnetosphere during magnetic storms (Yu and Ridley, 2013), although we have studied a quiet interval in SeptemberOctober 2007.

Another mechanism for Pc2-3 generation could be related to the minimum of the Alfven velocity under the plasmapause forming a waveguide for compressional (FMS) waves (Klimushkin, 1998). The existence of a second low-latitude 
maximum in the CHAMP Pc2-3 occurrence rate and PSD (Fig. 8) can be an indirect argument in favor of this hypothesis. A difference in wave polarization exists between waves inside and outside the plasmasphere. The amplitudes and occurrence rates for transverse $\left(b_{\varphi}, b_{R}\right)$ and field-aligned $\left(b_{\tau}\right)$ components in the outer magnetosphere $(L>4)$ are comparable. At lower $L$, they steeply decrease for $\left(b_{\tau}\right)$ so at $L<4$, the wave polarization is typical for shear Alfven waves. The azimuthal component also dominates in the $\mathrm{F}$ layer. Therefore Pc2-3s may be generated in the outer plasmasphere as FMS waves, and then they can be coupled/transformed into shear Alfven waves near the plasmapause (Fedorov et al., 1998; Mazur et al., 2001). The two maxima in the CHAMP Pc2-3 occurrence rate and PSD may correspond to two zones of minimal Alfven velocity: the first one is located under the plasmapause and the second one corresponds to low $L$ where the concentration of heavy ions of ionospheric origin is enough to reduce the Alfven velocity (Green et al., 1993).

The main result of the present study can be summarized as follows. Pc2-3 ( $f>70 \mathrm{mHz}$ ) pulsations are quite typical in the $\mathrm{F}$ layer of the Earth's ionosphere and in the magnetosphere. The amplitude and the occurrence rate of $\mathrm{F}$ layer Pc2-3s have maxima inside the plasmasphere and day-to-day variations of the Pc2-3 maximum position roughly repeat the plasmapause motion. Polarization and amplitude of Pc2-3s in the magnetosphere, in the ionosphere, and on the ground allow us to suggest that they are generated as FMS waves in the outer magnetosphere and are partly converted into Alfven waves near the plasmapause. The observed groundto-ionosphere amplitude ratio during the night may be interpreted as the result of the Alfven wave transmission through the ionosphere. The best agreement between the calculated and measured values of the ground-to-ionosphere amplitude ratio is found for $k=5 \times 10^{-3} \mathrm{~km}^{-1}$, i.e., the the observed ground-to-ionosphere amplitude ratio corresponds to a wave spatial scale which provides a Doppler shift within a few percent of the apparent frequency of the Pc2-3 pulsations as recorded by a low-orbiting spacecraft.

However, several experimental results have not been understood yet. The occurrence rates and amplitudes of the CHAMP Pc2-3 demonstrate an interhemispheric asymmetry (Fig. 8). This could be the result of seasonal variations of the ionospheric conductivity, and an analysis of longer time series than used in the present study is necessary to understand this effect. The other problem is related to the absence of the afternoon Pc2-3s on the ground surface (Fig. 9). Comparison of the model and observed ground-to-ionosphere amplitude ratios has shown a suitable agreement under realistic $k$ values in the post-midnight (MLT) sector, but no ground Pc23 has been selected by the automatic detection program in the afternoon sector. This effect could be related to diurnal variations of wave spatial scales and the resulting day-night contrast in ionospheric attenuation. Another reason may be a higher level of interference in the Pc2-3 range during the day. Again, an analysis of longer time series is necessary to discriminate between these two mechanisms. Probably, a recently started SWARM multi-satellite mission providing high-resolution magnetic and electric field measurements in the ionospheric $\mathrm{F}$ layer will lead to an accurate discrimination between different factors determining ULF parameters using the methods of automatic detection (Heilig et al., 2007; Balasis et al., 2013).

Finally, the most important problem concerns generation and propagation of Pc2-3 in the magnetosphere. In particular, a steep gradient of the Pc2-3 polarization in the magnetosphere near $L=4$ (Fig. 10) could be related to the plasmapause. This assumption should be checked by comparative analysis of Pc2-3s recorded simultaneously by several magnetospheric satellites.

Acknowledgements. The study was completed in the framework of a bilateral collaborative project based on an agreement between the Russian and the Hungarian Academy of Sciences and within the Program of Fundamental Studies no. 22 of the Presidium of the Russian Academy of Sciences (N. Yagova, E. Fedorov). The CHAMP mission was sponsored by the Space Agency of the German Aerospace Center (DLR) through funds of the Federal Ministry of Economics and Technology, following a decision of the German Federal Parliament (grant code 50EE0944). The authors thank H. Luehr for providing CHAMP data. We acknowledge NASA contract NAS5-02099 and V. Angelopoulos for use of data from the THEMIS Mission and specifically K. H. Glassmeier, U. Auster, and W. Baumjohann for the use of FGM data provided under the lead of the Technical University of Braunschweig and with financial support through the German Ministry for Economy and Technology and the German Center for Aviation and Space (DLR) under contract 50 OC 0302. The authors thank K. Pajunpaa (FMI, Finland), J. Reda (IGFPAS, Poland), V. Wesztergom (GGRI of HAS, Hungary), and their institutions for maintaining the MM100 network. The authors thank both referees for helpful criticism.

Topical Editor G. Balasis thanks M. Engebretson and one anonymous referee for their help in evaluating this paper.

\section{References}

Alperovich, L. and Fedorov, E.: The satellite electromagnetic sounding of the Earth, Adv. Space Res., 13, 23-32, doi:10.1016/0273-1177(93)90198-K, 1993.

Alperovich, L. S. and Fedorov, E. N.: Hydromagnetic Waves in the Magnetosphere and the Ionosphere. Series: Astrophysics and Space Science Library, Vol. 353, 2009, XXIV, 418 pp., Hardcover, ISBN: 978-1-4020-6636-8, 2007.

Balasis, G., Daglis, I. A., Zesta, E., Papadimitriou, C., Georgiou, M., Haagmans, R., and Tsinganos, K.: ULF wave activity during the 2003 Halloween superstorm: multipoint observations from CHAMP, Cluster and Geotail missions, Ann. Geophys., 30, 1751-1768, doi:10.5194/angeo-30-1751-2012, 2012.

Balasis, G., Daglis, I. A., Georgiou, M., Papadimitriou, C., and Haagmans, R.: Magnetospheric ULF wave studies in the frame of Swarm mission: a time-frequency analysis tool for automated de- 
tection of pulsations in magnetic and electric field observations, Earth Planets Space, 65, 1385-1398, 2013.

Bering III, E. A., Benbrook, J. R., Engebretson, M. J., and Arnoldy, R. L.: Simultaneous electric and magnetic field observations of Pc1-2, and Pc3 pulsations, J. Geophys. Res., 103, 6741-6761, doi:10.1029/97JA03327, 1998.

Bilitza, D.: International reference Ionosphere, Radio Sci., 36, 261275, 2001.

Budden, K. G.: Radio waves in the ionosphere, Cambridge University Press, Cambridge, 1966.

Carpenter, D. L. and Anderson, R. R.: An ISEE/whistler model of equatorial electron density in the magnetosphere, J. Geophys. Res., 97, 1097-1108, doi:10.1029/91JA01548, 1992.

Chatfield, C.: The analysis of time series, an introduction, 6th Edn., New York, Chapman-Hall/CRC, 352 pp., 2004.

Engebretson, M. J., Peterson, W. K., Posch, J. L., Klatt, M. R., Anderson, B. J., Russell, C. T., Singer, H. J., Arnoldy, R. L., and Fukunishi, H.: Observations of two types of Pc 1-2 pulsations in the outer dayside magnetosphere, J. Geophys. Res., 107, 1451, doi:10.1029/2001JA000198, 2002.

Fedorov, E., Mazur, N., Pilipenko, V., and Yumoto, K.: MHD wave conversion in plasma waveguides, J. Geophys. Res., 103, 2659526605, 1998.

Fraser, B. J., Samson, J. C., Hu, Y. D., McPherron, R. L., and Russell, C. T.: Electromagnetic ion cyclotron waves observed near the oxygen cyclotron frequency by ISEE 1 and 2, J. Geophys. Res., 97, 3063-3074, doi:10.1029/91JA02447, 1992.

Green, A. W., Worthington, E. W., Baransky, L. N., Fedorov, E. N., Kurneva, N. A., Pilipenko, V. A., Shvetzov, D. N., Bektemirov, A. A., and Philipov, G. V.: Alfven field line resonances at low latitudes ( $L=1.5)$, J. Geophys. Res., 98, 15693-15699, 1993.

Guglielmi, A. V. and Troitskaya, V. A.: Geomagnetic pulsations and diagnostics of the magnetosphere, Sov. Phys. Usp., 12, 195-218, 1969.

Heilig, B. and Lühr, H.: New plasmapause model derived from CHAMP field-aligned current signatures, Ann. Geophys., 31, 529-539, doi:10.5194/angeo-31-529-2013, 2013.

Heilig, B., Lühr, H., and Rother, M.: Comprehensive study of ULF upstream waves observed in the topside ionosphere by CHAMP and on the ground, Ann. Geophys., 25, 737-754, doi:10.5194/angeo-25-737-2007, 2007.

Heilig, B., Lotz, S., Verõ, J., Sutcliffe, P., Reda, J., Pajunpää, K., and Raita, T.: Empirically modelled Pc3 activity based on solar wind parameters, Ann. Geophys., 28, 1703-1722, doi:10.5194/angeo28-1703-2010, 2010.

Hughes, W. J. and Southwood, D. J.: The screening of micropulsation signals by the atmosphere and ionosphere, J. Geophys. Res., 81, 3234-3240, doi:10.1029/JA081i019p03234, 1976.

Kay, S. M.: Modern spectral estimation - Theory and application, Prentice-Hall, 543 pp., 1988.

Klimushkin, D. Y.: Resonators for hydromagnetic waves in the magnetosphere, J. Geophys. Res., 103, 2369-2375, doi:10.1029/97JA02193, 1998.
Klimushkin, D. Yu., Mager, P. N., and Glassmeier, K.-H.: Axisymmetric Alfvén resonances in a multi-component plasma at finite ion gyrofrequency, Ann. Geophys., 24, 1077-1084, doi:10.5194/angeo-24-1077-2006, 2006.

Knudsen, D. J., Kelley, M. C., and Vickrey, J. F.: Alfvén waves in the auroral ionosphere: A numerical model compared with measurements, J. Geophys. Res., 97, 77-90, doi:10.1029/91JA02300, 1992.

Le, G., Chi, P. J., Strangeway, R. J., and Slavin, J. A.: Observations of a unique type of ULF wave by low-altitude Space Technology 5 satellites, J. Geophys. Res., 116, A08203, doi:10.1029/2011JA016574, 2011.

Leonovich, A. S. and Mazur, V. A.: An electromagnetic field induced in the ionophere and atmosphere by low frequency Alfven oscillations of the magnetosphere: general theory, Planet. Space Sci., 39, 529-546, 1991.

Mazur, N. G., Fedorov, E. N., and Pilipenko, V. A.: Emission of Alfven waves from a nonuniform MHD waveguide, Plasma Phys. Rep., 27, 773-784, 2001.

Mikhailova, O. S.: The spatial structure of ULF-waves in the equatorial resonator localized at the plasmapause with the admixture of the heavy ions, J. Atmos. Sol.-Terr. Phy., 108, 10-16, 2014.

Nishida, A.: Ionospheric screening effect and storm sudden commencement, J. Geophys. Res., 69, 1861-1874, 1964.

Nose, M., Takahashi, K., Anderson, R. R., and Singer, H. J.: Oxygen torus in the deep inner magnetosphere and its contribution to recurrent process of O+-rich ring current formation, J. Geophys. Res., 116, A10224, doi:10.1029/2011JA016651, 2011.

Sciffer, M. D. and Waters, C. L.: Propagation of ULF waves through the ionosphere: Analytic solutions for oblique magnetic fields, J. Geophys. Res., 107, 1297, doi:10.1029/2001JA000184, 2002.

Sciffer, M. D., Waters, C. L., and Menk, F. W.: Propagation of ULF waves through the ionosphere: Inductive effect for oblique magnetic fields, Ann. Geophys., 22, 1155-1169, doi:10.5194/angeo22-1155-2004, 2004.

Sciffer, M. D., Waters, C. L., and Menk, F. W.: A numerical model to investigate the polarisation azimuth of ULF waves through an ionosphere with oblique magnetic fields, Ann. Geophys., 23, 3457-3471, doi:10.5194/angeo-23-3457-2005, 2005.

Singh, N. and Horwitz, J. L.: Plasmasphere refilling: Recent observations and modeling, J. Geophys. Res., 97, 1049-1079, doi:10.1029/91JA02602, 1992.

Yagova, N., Heilig, B., Fedorov, E., Kovacs, P., Luhr, H., Reda, J., Pajunpaa, K., and Raita, T.: Pc2-3 geomagnetic pulsations on the ground and in space. Results of comparative amalysis of MM100 and CHAMP data, Abstract book of the IAGA 11th Scientific Assembly (Sopron, 24-29 August, 2009), 310-SAT-P1703-1002, 2009.

Yu, Y. and Ridley, A. J.: Exploring the influence of ionospheric O+ outflow on magnetospheric dynamics: The effect of outflow intensity, J. Geophys. Res.-Space, 118, 5522-5531, doi:10.1002/jgra.50528, 2013. 